\title{
L'Integrazione, Il Mercato Comune e L'Unificazione del Diritto Dell'America Latina.
}

\author{
R. Limongi França \\ Professore della Facolta di Diritto della \\ Universita di São Paulo.
}

SOMMaRIo: I - IL DIRITTO INTERNAZIONALE E LE Comunità Plurinazionali - 1. Oggetto del Diritto Internazionale. 2. Evoluzione del Diritto Internazionale. 3. Le comunità plurinazionali. II. IL DIRITTo INTERNAZIONALE E L'UNIFICAZIONE DEL DIRITTO 1. Il Diritto consuetudinario internazionale unificato. 2. Avventa dello Stato Moderno e determinazione del Diritto Privato. 3. Il ritorno al diritto unificato. III. L'INTEGRAZIONE DELL'AMERICA LATINA $e$ L'UNIFICAZIONE DEL DIRITTO AD ESSA APPARTENENTE 1. T'endenza dell'America Latina all'internazionalità.

2. Evoluzione dell'Integrazione dell'America Latina

3. Organismi Integrativi dell'America Latina. 4. La unificazione del Diritto come substrato dell'integrazione dell'America Latina. IV. L'INTEGRAZIONE DEL DiRItTo DELle OBbligazioni E DEI CONTRATTI DELL' AMERICA LATINA - 1. La presenza nel diritto universale del Diritto dell'America Latina. 2. Affinità sostanziali del Diritto dell'America Latina. 3. Le radici del Diritto Privato dell'America Latina. 4. Il Diritto delle Obbligazioni e dei Contratti dell'America Latina. V. Prospettive Circa UN'Unificazione Universale DEL DIRITTO - 1. L'America Latina e l'America Anglo-Sassone. 2. L'America Latina e l'Europa. 3. L'America Latina e l'Oriente. 4. L'America Latina e il Mondo Africano. VI. CoNCLUSIONE - 1. Visione d'insieme. 2. Fallimento delle unificazioni europee preterite. 3. L'America Latina come il cammino più facile per il raggiungimento dell' unificazione del Diritto. 4. L' unificaziono del Diritto delle Obbligazioni e dei contratti dell'America Latina come punto di partenza. 
I. II Diritto Internazionale e le comunità plurinazionali.

\author{
1.Oggetto del Diritto Internazionale. 2. Evoluzione \\ del Diritto Internazionale. 3. Le comunità plurina- \\ zionali.
}

1. Oggetto del Diritto Internazionale. Bustamante insegna, secondo la traduzione di CouLÉ, che il Diritto Internazionale è — "l'ensemble des principes qui règlent les droits et les devoirs extéricurs et les relations des personnes juridiques qui font partie de la communauté internationale, entre elle et avec la Société des Nations et l'Union Panaméricaine, ainsi que les règles communes de protection individuelle interne ou externe établies par accords internationaux. (Droit International Public, I, page 3, 1934).

Sebbene questa definizione rimonti a parecchi anni fa, essa è tuttora valida, sempre che le espressioni "Società delle Nazioni" e "Unione Panamericana", siano sostituite da altre corrispondenti, ma più attuali, cioè "Organizzazione delle Nazioni Unite" (oNU) e "Organizzazione degli Stati Americani" (OEA), aggiungendovi, inoltre, un riferimento alle istituzioni plurinazionali.

Dal concetto promana il proprio oggetto del Diritto Internazionale, ciọe il Bene Comune della società degli stati e dei relativi cittadini, in quanto partecipanti a detta società.

Quanto all'espressione "Bene Comune" non è questa un'idea da collocare su un piano semplicemente teorico, per servire come tema a filosofi ne a filologi. Si tratta piuttosto di qualcosa che, nel piano interno degli stati e in modo particolare in quello delle comunità delle nazioni, è direttamente e immediatamente relazionato con le regole e le possibilità della convivenza; della facilità delle relazioni umane; del dialogo costante; dell'intercambio di utili; del 
superamento di quegli ostacoli che sono causa di guerra; del perfezionamento degli elementi auspicatori una pace che possa diventare a poco a poco completa; di tutto quanto serva, a breve o lunga scadenza, al conseguimento, sul piano internazionale, delle finalità integrali dell'Uomo.

2. Evoluzione del Diritto Internazionale. A noi sembra che, coscientemente o no, solamente questa sia stata la molla propulsatrice della Storia del Diritto Internazionale.

Così è stato fin dai primi trattati di pace firmati da popoli antichissimi, come si può costatare osservando $i$ testi cuneiformi assiro-babilonesi.

Nello stesso modo, nella Grecia antica, si ha il sorgere dell'arbitrato, come soluzione delle liti, l'inviolabilità dei messaggeri, il diritto di asilo, l'intercambio dei prigionieri, e altre numerose istituzioni simili.

A Roma, nonostante l'esistenza del Jus Feciale, si è potuto costatare un minor sviluppo del Diritto Internazionale (Hildebrando Accioly, Manual de Direito Internacional Público, pg. 15. 1948); ciò va attribuito al fatto che, nell'Orbs Romanus, considerate le circostanze, createsi soprattuto dopo la costituzione di Antonio e Caracalla (212 a.C.) destinata alla generalizzazione della cittadinanza, la struttura politica dell'impero riposava sulle relazioni pacifiche tra i popoli (Arangio - Ruiz, Historia del Derecho Romano, 1943).

Durante il basso medioevo, più che il vero e proprio desiderio, fu la necessità della convivenza internazionale a generare sia l'unione tra i goti e i cristiani, consolidata da Recaredo, nel Concilio di Toledo del 589 (Gama Barros, História da Administração Pública em Portugal, 2. ${ }^{a}$ ed. 1945); Antonio Matroso, História da Civilização, 3. ${ }^{\mathrm{a}}$ ed., s/d); che il modus vivendi moro, dalla caduta di Witisa (711) fino alla fine del dominio mussulmano.

Non diverso fu il carattere della Pace di Dio e della Tregua di Dio, durante l'agitato ed eroico periodo delle Crociate. 
Da quei tempi in avanti, qualunque dimostrazione $\grave{e}$ superflua.

I fatti e la scienza si sono dati la mano nella ricerca di norme capaci di cementare la sicurezza e il progresso della vita umana, sempre più vertiginosamente complessa.

Da un lato, il commercio con l'oriente, le grandi navigazioni, la scoperta dell America, la fine del feudalesimo, l'apparizione dello Stato Moderno; dall'altro l'opera di Vitoria, Suarez, Grotius, Pufendorf.

La stessa guerra divenne un veicolo di pace, a volte con le condizioni favorevoli per essere più durevole -: Westralia 1648), con il principio dell'uguaglianza giuridica degli Stati; il Congresso di Vienna (1814-1815) con il nuovo ordine europeo post-napoleonico; e nuovamente, la presenza provvidenziale dell'America, come fattore di equilibrio internazionale, attraverso l'indipendenza delle colonie spagnole e portoghesi, che rese possibile la formazione, non solo dei paesi dell'America Latina, ma dello stesso terzo mondo; ne furono sintomi dei fatti importanti verificatisi durante il periodo che va dalla Dichiarazione di MONRoE, (1823) alla Prima Conferenza Interamericana (1889).

In seguito, la prima Guerra Mondiale e la Società delle Nazioni; la seconda Guerra Mondiale e l'Organizzazione delle Nazioni Unite.

3. Le Comunità Plurinazionali. Senza alcun dubbio, l'esperienza post-bellica generò, tra i suoi frutti più rilevanti, la formazione a carattere ufficiale, definitivo e inequivocabile, delle comunità plurinazionali, nei termini del capitolo vin della Carta delle Nazioni Unite, sotto la voce "Accordi Internazionali".

Di codesto capitolo, è opportuno mettere in risalto l'art.

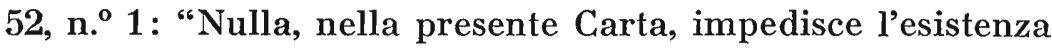
di accordi o di entità regionali, destinati a trattare questioni relative alla conservazione della pace e della sicurezza internazionali, suscettibili di un'azione regionale, sempre che 
tali accordi o entità regionali e relative attività, siano compatibili con i Propositi e i Principi delle Nazioni Unite".

A sua volta, il Cap. Ix, riferentesi alla "Cooperazione Economica e Sociale Internazionale", sebbene l'ar. 57 n. ${ }^{\circ} 1$ parli della vincolazione alle Nazioni Unite, da parte delle entità destinate a prendersi cura del settore "economico, sociale, culturale, educativo, sanitario e simili", è a tal punto vago da non impedire, come difatti non ha impedito, che codesti organismi venissero a trovarsi nella condizione di subordinazione stabilita attraverso le strutture regionali.

Cosicchè, sia sul piano internazionale che su quello plurinazionale, hanno proliferato le comunità intermediarie.

Un esempio delle prime è la ITo (International Trade Organization), i cui negoziati iniziali ebbero luogo a Londra (1946), proseguirono a New York e a Ginevra (1947) e si formalizzarono all'Havana (1948). Sorta da concezioni propagate da CoRdell Hull, secondo le quali "l'eliminazione di ostacoli commerciali tra i paesi porterebbe non solo ad una maggior efficienza economica e a un maggior benessere internazionale, ma assicurerebbe, altresi, le condizioni essenziali ad una pace mondiale duratura, (Celso LAFER, $O$ Gatt, A Cláusula de Nação Mais Favorecida e a América Latina, nella Rivista del Diritto Mercantile, n. 3 - 1971), essa finì col non essere ratificata dagli Stati Uniti e perse, quindi, le condizioni politiche necessarie alla sua completa vigenza.

Ciò nonostante, tuttavia, da essa provenne la creazione del gatT (General Agreement on Tariffs and Trade), che senza dubbio, più debolemente, abbraccia una parte considerevole delle norme di politica commerciale negoziate nella Carta dell'Havana.

Quanto alle comunità intermediarie regionali, bisogna considerare la parte decisiva che, come è noto, esse hanno, per esempio, nella Comunità Europea; le informazioni relative sono state magnificamente esposte ed ordinàle, 
da W. J. Ganshof Van Der Meersch, illustre professore della Facoltà di Diritto di Bruxelles, nella sua opera ormai classica, Organizations Europeénnes (Bruxelles, Paris, 1966).

Infatti, gli specialisti e la stessa opinione pubblica internazionale non disconoscono le rilevanti funzioni del COMEcoN (Conseil d'Assistence Economique Mutuelle, Mosca, 1949); del Patto di Varsovia (1955); della otan (Organization du Traité de l'Atlantique Nord, Washington, 1949); del Consiglio d'Europa 1948 - 1949), con sede a Strasbourg); del Benelux (1. ${ }^{\circ}$ Gennaio del 1948); della ocDE (Organization de Coopération et de Développement Economique, Paris, 1960); della EFTA o AELE (European Free Trade Association - Association Europeénne de Libre Échange, Stockholm, 1960) e, soprattutto, del Mercato Comune Europeo, sempre più operante.

\section{Il Diritto Internazionale e l'unificazione del Diritto.}

1. Il Diritto Consuetudinario Internazionale Unificato.

2. Avvento dello Stato Moderno e determinazione del

Diritto Privato. 3. Il ritorno al diritto unificato.

1. Il Diritto Consuetudinario Internazionale Unificato. Indubbiamente, una delle più importanti considerazioni che si trovano nel Rapport sur le projet d'un code des obligations et Contrats commun da la France et $\dot{a}$ l'Italie, sottoscritto da maestri eminenti come Capitant e Ruggiero, è quella secondo la quale - "l'uniformité du droit privé entre les Etats les plus civilisés est un problème qui s'impose, par suite de la nécessité de cette Communitas gentium, toujours plus en progrès dans les civilizations modernes", come quella de "cette uniformité fut, au moins pour le droit des obligations, une realité historique pendant des siècles en Europe" (pages xxII - xxv, Roma, 1928). 
Questa osservazione, tuttavia, comporta una ampiezza ancor maggiore.

Certamente, durante $\mathrm{i}$ periodi storici più antichi, dovette esistere una certa identità di principi procedenti dal proprio Diritto Naturale, e a poco a poco discriminati dal Diritto delle Genti; essa rese possibile il commercio dei Fenici, degli Stati Greci tra di loro e con gli altri popoli, delle popolazioni dell'Impero Romano unificato, e posteriormente delle nazioni europee, specialmente di quelle città - stato che, come Genova e Venezia, trafficavano con l'Oriente, ancor prima del tempo delle grandi navigazioni.

E inutile dire che il maggiore o minore sviluppo tecnico dipese dalle circostanze economico-politiche le più diverse, cosicchè si riscontrarono variazioni che andarono dal più elementare empirismo dell'intercambio diretto alla raffinatezza dei contratti stipulati secondo il sistema romano.

Con lo sviluppo del commercio marittimo, elementi altamente significativi si indirizzarono verso l'unità del Diritto delle Obbligazioni e dei Contratti, come si può costatare osservando certe compilazioni che consolidarono il modus vivendi delle nazioni e delle comunità di tipo commerciante.

Tra queste risalterebbero la Tabula de Amalfi (secolo x, XI o XII), Rôles d'Oléron, (secolo XII) e, sopratutto, il Consolato del Mare (secolo XIV). Sebbene legate specificamente al Diritto Marittimo, codeste compilazioni si collegano strettamente allo stylus mercatorum, che non era altro, in fondo, che il diritto commerciale dell'epoca (e quindi delle obbligazioni e dei contratti), con carattere indiscutibilmente unitario (v. LatTes, Il Diritto Commerciale nella Legislazione Statutaria delle Città Italiane, pagina 23, Milano, 1884; Vivante, Trattato, I, pe. 2 a 5, 5.i ed.).

2. L'Avvento dello Stato Moderno e la determinazione del Diritto Privato. Con l'avvento dello Stato Moderno, di cui il regno di D. João II in Portogallo (v. Eduardo D'Oliveira 
França, $O$ Poder Real em Portugal $e$ as Origens do Absolutismo, U.S.P., 1946), fu certamente una delle prime, se non la prima, grande pietra miliare, e la cui definitiva affermazione fu ratificata dai Trattati di Westralia (Cf. Dalmo Dallari, Elementos de Teoria Geral do Estado pg. 62, Saraiva 1972), esistette un provvedimento, costantemente preso come manifestazione di sovranità : l'organizzazione interna del Diritto Privato.

In un certo senso, ciò si era già fatto sentire in Portogallo, nel 1446, in occassione della promulgazione delle "ordinanze" di D. Alfonso V.

Ma fu solo dopo la Guerra dei Trent'Anni che troviamo, nell'Europa civilizzata, i grandi codici interni, da quello della Norvegia e della Dinimarca (1863), a quello della Svezia (1734), a quello Bavarese del 1756 (Codice Massimiliano) e a quello Prussiano del 1794 (Codice Federico). Bisogna far notare la grande importanza che ebbero gli studi preliminari del Codice Austriaco, intrapresi, sotto il regno di Maria Teresa, da una Commissione istituita nel 1753, e che servirono come sussidio al proprio Codice Napoleonico del 1803 (v. Mrchel Doucer, Code Civil Général Autrichien, Avant-propos, page 7, Paris, 1947).

A partire da questo codice, pubblicato sette anni dopo (1810), tutte le nazioni europee regolorano il loro diritto privato interno o su di esso o su quello Austriaco.

Cosi, per esempio, nello stesso 1838, mentre la Sardegna promulgava un regolamento ispirato al codice napoleonico, l'Olanda ne emanava un'altro secondo il modello ausiriaco.

Nel frattempo, l'America Latina acquistava la sua indipendenza e tutte le nazioni del Terzo Mondo si preoccuparono, da quel momento in poi, di codificare il loro Diritto Privato. In Brasile, ancor prima della prima costituzione del 1824, era stato promesso il Codice Civile con una legge speciale risalente al 1823. (v. Limongi França, Manual de Direito Civil, vol. I, pag. 99, $2 .^{\text {a }}$ ed., 1971; dello stesso autore, $O$ Có- 
digo Civil Brasileiro, 1967; Candido Mendes de Almeida, Codice Filippino, Rio, 1870).

3. Il Ritorno al diritto unificato. Capitant, Ruggiero e gli altri collaboratori del Rapport (Relazione) del Progetto Franco-Italiano, sottoscrissero l'affermazione che "Le Code Napoléon, tout en contribuant à détruire ce règne du droit commun, fut, cependant, le point de départ à une nouvelle unification" (pages xxiv, xxv).

Questa affermazione è esatta, ma deve essere completata.

Infatti, sebbene la maggior parte dei paesi dell'Europa e dell'America Latina abbia usato come base il modello francese, per lo meno tre osservazioni devono essere fatte in proposito. Innanzi tutto, il modello napoleonico è, più che francese, e prima di francese, sostanzialmente latino, nel senso che rappresenta una cultura molto più ampia e molto più antica.

In secondo luogo, secondo una prospettiva più prossima, il modello francese ha in sè elementi di ispirazione germanica, in virtù dell'influenza dei lavori del Codice Austriaco e della stessa giurisprudenza nordica, di rilevatissima importanza in tutto il diritto dell'epoca.

Terzo: molti paesi, quando stabilirono il loro diritto privato interno, non seguirono il modello francese, ma quello tedesco.

Un esempio realmente notevole ci viene offerto dal Codice del Giappone, che, ordinato a Borssonade nel 1879, non potè essere realizzato a causa del suo rigido "gallicismo"; fu necessario sostituirlo, nel 1898, con un altro di tipo germanico "The books on general provisions - ci dice SEBald - real rights, and obligations are predominantly german" (The Civil Code of Japan).

Avviene peraltro che lo stesso Diritto Germanico, come scienza e prospettiva, ha un carattere sostanzialmente latino, tanto che è proprio in questo punto di convergenza dei sis- 
temi francese e tedesco che risiede la pietra angolare del ritorno al diritto unificato, poichè sia l'uno che l'atro, pur senza smentire l'origine comune, hanno influito sul Diritto Occidentale, su quello del Paraguai, dell'Argentina e del Brasile e su quello orientale, come si può vedere leggendo il Codice Civile della Cina di Sun Yat Sen (v. Code Civil de la République de Chine, ed. di Ho Tchong T-Chan, Changai, Paris, 1930-1931.

Sicuramente, oltre alle ragioni politiche determinanti, fu questa la base istituzionale che rese possibile l'unificazione del Diritto delle Obbligazioni in Svizzera, attraverso il Code Féderal des Obligations, promulgato nel 1882 e rinnovato nel 1911; nonostante le origini e la cultura latine e germaniche di codesto paese, le sue disposizioni sono "applicables aux autres matières du droit civil" (v. FriTz Funk, Commentaire du Code Fèderal des Obligations, trad. de Porret e Perregaux, Neuchatel, 1930).

In seguito sorge il Progetto Franco-Italiano, concepito da Scialoja durante la prima Grande Guerra; egli lo espose nella Nuova Antologia, il I febbraio del 1926, venendo appoggiato da Larnaude, Decano della Facoltà di Diritto a Parigi. Ne derivò come conseguenza la nomina di due commissioni, una italiana e l'altra francese, sotto la presidenza del citato maestro peninsolare. Risultato dei suoi Iavori, portati a termine con alto spirito civico e scientifico, fu il testo del 31 ottobre del 1927, comprendente 739 articoli.

Sebbene, secondo Consentrini, codesta opera fosse stata "une déception pour le monde juridique", fu proprio essa che lo ispirò e gli servì come base per il Progetto del Code International des Obligations, con 3115 articoli, divulgato dall'Institut Américain de Droit et Legislation Comparée, di Berna, ben più vasta per ambizione, poichè fu realizzata "en vue de l'unification des législations civiles et commerciales sur des bases internationales". 
Non tardò ad esplodere la II Grande Guerra e, come abbiamo visto, il mondo della seconda metà del secolo $\mathrm{XX}$, sofferente sotto il colpo non mai provato della conflagrazione universale, cercò di risorgere su delle basi le più solide possibili di sopravvivenza e convivenza; si incentivò il progresso delle comunicazioni, si intensificò il dialogo e si crearono infrastrutture intermedie attraverso organismi plurinazionali e regionali.

Questa nuova e progredita mentalità universale favori, come mai anteriormente, il ritorno al diritto Unificato.

\section{L'integrazione dell'America Latina e Punificazione del suo Diritto.}

1. La tendenza dell'America Latina all'internazionalità.

2. Evoluzione dell'integrazione dell'America Latina.

3. Organismi integrativi dell'America Latina. 4.

L'unificazione del Diritto come sottostrato giuridico dell'Integrazione dell'America Latina.

1. Tendenza dell'America Latina all'Internazionalità. Non vi è esagerazione od enfasi quando si parla di una tendenza all'Internazionalità da parte dell'America Latina.

In verità, essa si verifica nel campo che ben potremmo chiamare di storia interna ed esterna.

Quanto alla prospettiva estera, è generalmente noto, e tra gli altri Verdososs ne è testimone, che la scoperta dell'America e la colonizzazione del Nuovo Mondo da parte di popoli europei, specialmente Spagna e Portogallo, "trouxe consigo uma grande dilatação do âmbito espacial do Direito Internacional Cristão - Europeu" (Derecho Internacional Publico, 4. ${ }^{a}$ ed., trad. esp. del 1967, pag. 41).

$\mathrm{E}$ ancor prima che fossero riconosciuti come soggetti di Diritto Internazionale i grandi stati asiatici, come l'Impero del Giappone, dopo la sua vittoria sulla Russia nel 1905, e 
in seguito la Cina, il Siam (Tailandia) l'Afganistan e la Persia, l'America Iberica, vale a dire l'America Spagnola e Portoghese, l'America Latina, insomma, grazie alla proclamazione dell'indipendenza delle colonie ormai maturate circa un secolo prima, col riconoscimento delle rispettive sovranità, aveva già allargato gli orizzonti del Diritto Internazionale, vale a dire della comunità del popoli, favorendo inoltre il dialogo con l'Oriente stesso.

E già nel 1823 questa America Latina, posta dinanzi alla politica intervenzionista della cosiddetta Santa Alleanza, rappresentò, per la prima volta come blocco comunitario, un fattore decisivo di equilibrio universale, suscitando la dichiarazione di Monroe.

Da questo momento in poi, e sopratutto, dalla Prima Conferenza Interamericana realizzata a Washington dall'ottobre del 1889 all'aprile del 1890, l'America Latina fu presente alle sedute durante le quali furono presi innumerevoli, decisivi provvedimenti che assicurassero la pace, la concordia e la comunicazione universale. Così avvenne, per esempio, alla Conferenza del Messico (1901-1902), alle Convenzioni dell'Avana del 1928, alla Conferenza Straordinaria di Buenos Aires del 1967.

Dal punto di vista interno, si riscostrano le prime radici ben nitide già durante il Basso Medioevo, particolarmente quando Recabedo, nel primo Concilio di Toledo (589) realizzò la confraternizzazione Gotico-Romana; questo, se da un lato costitui, nel consolidamento delle basi della Cultura Iberica, un fondamento del mondo latino-americano, dall'altro trasformò la Penisola nel centro universale della latinità stessa, e politicamente parlando, della stessa Cristianità.

Di lì sorsero le grandi e decisive azioni contro i Mori e, dai trionfi della Riconquista, le strade del Nuovo Mondo che è poi l'America Latina dei nostri giorni.

2. Evoluzione del'Integrazione dell'America Latina. Quando, agli albori dell'America, Cortez penetrava nel Cä- 
ribe e nel Messico, Pizarro sulle coste del Pacifico e Cabral, seguito da Martim Afonso, nelle terre dell'Atlantico, essi stavano già tracciando, in un ambito storicamente innegabile, le basi rudimentali dell'integrazione dell'America Latina.

E non si tratta di un lirismo ancestrale, nè della risonanza del poema di Ercilla o delle Brasileidi di Carlos Alberto Nunes.

È che, in verità, i nuclei della nuova civiltà che tre secoli dopo si sarebbero sviluppati come terzo mondo, portavano già in sè il componente comune della cultura latino-goticamora-cristiana, che, ancora ai nostri giorni, rappresenta la tonica fondamentale della nostra mentalità.

Divenne ancora più pressante, questa realtà, con il disastro di Alcacer-Quibir, la morte di D. Sebastiano deI Portogallo (1578), la morte, immediatamente successiva del Cardinale D. Eurico, suo successore (1580) e l'unione con la Spagna durante il regno dei Filippi. In questo periodo si verificò un fatto, tra i molti che avvennero, definitivamente integrativo: la promulgazione, nel 1603, delle ordinanze Filippine. Per avere una idea definitiva della loro importanza in questo campo, basta ricordare che, pur essendo una legge spagnola, rimase in vigore in Brasile fino al 1..$^{\circ}$ Gennaio del 1917.

Non vi è alcun dubbio che, quando più tardi, durante il secolo xIx, le colonie spagnole e portoghesi andarono gradatamente proclamando le rispettive indipendenze, il movimento separatista di ognuna di esse costitui un incentivo, una base ideologica, politica ed anche economica per la liberazione di tutte le altre colonie, formò, anzi, l'infrastruttura sociale e politica della Dichiarazione di Monroe e della conferenza internazionale del 1889.

3. Organismi Integrativi dell'America Latina. L'integrazione dell'America Latina è una conseguenza naturale dell'origine comune, della cultura comune, degli ideali co- 
muni, delle prospettive comuni circa il futuro dei suoi popoli, nell'ambito della società internazionale e della storia.

Così, diversi organismi plurinazionali si sono costituiti in virtù delle imposizioni di codesta realtà.

Dentro questa prospettiva persino alcune convenzioni più ristrette, come il trattado di Diritto Internazionale Privato dell'Equatore con la Colombia, del 18 giugno del 1903, tuttavia in vigore (v. Recueil $105-128,1968$, pg. 194; Haroldo Valladão, Direito Internacional Privado, testi, $5 .^{\text {a }}$ ed., pag. 278 e seg.), hanno disimpegnato una parte ben chiara e sempre più positiva.

Ancora più espressivi per l'estensione del numero di paesi che comprendono e per la quantità di interessi che collimano, sono i movimenti e gli accordi dell'America Centrale, ispirati a Bolrvar. Dalla Carta di São José (1951), dalla quale scarturì l'organizzazione degli Stati del Centro America (ODECA), essi culminarono, nel 1960, col trattato generale dell'Integrazione Economica dell'America Centrale. Il Patto Andino puó rappresentare un'altro esempio, a sostegno di quanto é già stato detto.

Tuttavia, il grande fulcro della marcia verso l'integrazione dell'Americana Latina, è innegabilmente l'ALALC (Associazione dell'America Latina del Libero Commercio), creata dal trattato di Montevideo il 18 febbraio del 1960.

Nel capitolo II, sotto la voce Programma della Liberazione dell'Intercambio, le parti contraenti si proposero di eliminare gradualmente "per i settori essenziali del commercio reciproco, tutti quei tipi di gravami e di restrizioni che possono incidere sull'importazione di prodotti originari dal territorio di qualsiasi Parte contraente".

In questo modo, si stabiliscono, tra gli altri elementi d'integrazione, sempre nei limiti di una visione realista e fattibile della convivenza contemporanea, le basi per un Mercato Comune dell'America Latina. 
4. L'unificazione del Diritto come sostrato dell'integrazione dell'America Latina. Di conseguenza, si mette in evidenza da solo il fatto che tutta l'integrazione della comunità, non solo nel campo economico, ma anche in quello politico e sociale, dipende in gran parte dall'integrazione giuridica.

Questa integrazione giuridica deve operare in due sfere: una interna e una esterna.

Nell'esterna, attraverso i trattati, le convenzioni, i protocolli e altri atti costitutivi degli organismi plurinazionali e nazionali.

Nell'interna, mediante l'unificazione del rispettivo Diritto Privato.

Indiscutibilmente, innumerevoli ostacoli, quasi intrasponibili, sorgerebbero se si arrivasse all'unificazione dello stato giuridico di certe istituzioni, profondamente radicate nelle tradizioni locali, come, per esempio ,la Famiglia.

Ma una tale situazione non si verifica per quanto riguarda il Diritto delle Obbligazioni e dei Contratti. Bisogna quindi considerare tanto le naturali possibilità di questo settore, quanto la sua impostergabile necessità come fattore di superamento previo di conflitti, nelle questioni giuridiche della comunità economica.

\section{L Integrazione del Diritto delle Obbligazioni e dei Contratti dell'America Latina.}

1. La presenza del Diritto dell'America Latina nel Diritto Universale. 2. Affinità sostanziale del Diritto dell'America Latina. 3. Le radici del Diritto Privato. dell'America Latina. 4. Il Diritto delle Obbligazioni e dei Contratti dell'America Latina.

1. La presenza del Diritto dell'America Latina nel Diritto Universale. L'importanza di questa materia è stret- 
tamente collegata alla valutazione del grado di maturità del Diritto dell'America Latina, poichè non avrebbe senso cercare di unificare qualcosa che mancasse di un previo sviluppo intrinseco.

In verità, nonostante le origini a volte legate ad ordinamenti stranieri, la Dottrina e la Legislazione dell'America Latina, dovuto alla solida formazione e cultura di molti suoi giuristi, come ANdres Bello, cileno, Velez Sarsfield, argentino, Teixerra de Freitas, brasiliano, e tanti altri, si sono solidamente affermate, tanto da influire anche sul Diritto Europeo e Orientale.

Basterebbe l'esempio del Codice di Bustamante, geniale maestro cubano, per mettere in evidenza la presença del Diritto dell'America Latina nel sistema giuridico universale; infatti, nonostante il ristretto ambito della Conferenza dell' Avana del 1928, questo testo esemplare è stato invocato per risolvere pendenze di paesi non firmatari.

Questa prospettiva ,tuttavia, acquista un rilievo altamente notevole, quando vediamo il nostro Diritto Privato influire sulla formazione del Diritto Privato di paesi dalla storia molto più antica della nostra.

E quello che avvenne, per esempio, con il Codice della Cina Repubblicana del 1929. Nella prefazionē dell'edizione ufficiale in francese elaborata dal presidente della Commissione Codificatrice, Ping Sheung Foo, accanto alla menzione del Codice Svizzero delle Obbligazioni e del Codice Sovietico della Famiglia, esiste il riferimento specifico al Codice Brasiliano del 1916 (pag. xxv).

$\mathrm{E}$ non è questo un caso isolato.

Quando le Commissioni Italiana e Francese risolsero di portare a termine il piano Scialoja e Larnaude, affinchè i loro rispettivi paesi potessero usufruire del sopra citato progetto unico del Codice delle Obbligazioni, non omisero questa preziosa testimonanza nel Rapport (Relazione) preliminare; "C'est ainsi que nous avons au cours de nos travaux durant le brulant conflit de la guerre pris en considération 
les plus récentes d'Europe et D'Amérique, du code de l'empire allemand de 1900 Au Code BrésILIEN de 1916, des nouvelles qui ont reformé le code autrichien pendant la guerre, au code suisse des obligations dans son texte révisé de 1911, du projet du code hongrois jusq'au code tout récent des republiques soviétiques" (pgs. xxxviII - xxxIx).

Ancora più significativo è ciò che si può leggere nel Code International des Obligations del Prof. Cosentini.

Le sue dichiarazioni sono state riportate nella Prefazione, a conferma della nostra tesi.

Infatti, mentre già nella prima pagina dichiara che la sua "grande oeuvre comparative a commencé en vue d'une prémière unification du droit privé latino-américain", in seguito mette in risalto $i$ progressi fatti dalla materia delle Obbligazioni in relazione al codice Napoleonico, grazie agli ordinamenti di varie nazioni, tra le quali "tous les codes de l'Amérique Latine" (pag. 7).

In tutto il testo, composto daì 3115 articoli del Progetto, ricorre frequentemente la citazione esplicita di questi codici.

Tanto per darne un esempio, basta notare che il concetto di contratto adottato dal progetto di Codice Internazionale delle Obbligazioni è ispirato dai seguenti documenti dell'America Latina: Codice dell'Argentina, art. 1171; della Bolivia, art. 692; del Cile, art. 1438; della Colombia, art. 1495; del Guatemala, art. 1365; delle Honduras, art. 1539; del Messico, art. 1272; del Nicaragua, art. 1365; del Panama, art. 1105; del Perù, art. 1226; del Salvador, art. 1327; dell' Uruguai, art. 1247; del Venezuela, art. 1174; a sua volta, ogni statuto della mora, ha le sue radici nel Codice Argentino, art. 563; in quello Brasiliano, artic. 955 e 1 a 58; in quello Cileno, art. 1551; in quello Colombiano, art. 1608; in quello dell'Honduras, art. 1355; in quello Messicano, artic. 1423, 1432 e 1439; in quello del Nicaragua ,art. 1859, in quello del Panama, art. 985, in quello del Salvador, art. 1440 ecc. ecc. 
2. Affinità sostanziale del Diritto dell'America Latina. Quando, nel capitolo III, n. $^{\circ} 2$, abbiamo trattato dell'evoluzione dell'integrazione dell'America Latina, sono state illustrate, anche, in un certo modo, le basi dell'affinità del Diritto rispettivo.

Tuttavia, a questo punto, possiamo e dobbiamo riprendere la questione in modo più sistematico.

Effettivamente questa affinità si fonda sulle seguenti basi primordiali: A) base latino-romanica; B) base cristiana; c) base gotico-mora; D) base canonica; E) quella che potremmo chiamare "iberica" in senso stretto.

Chiamiamo latino-romanico il componente culturale e giuridico proveniente non soltanto dal Grande Impero, ma da tutti i fattori che gli dettero la possibilità di sorgere e fiorire: dalle culture arcaiche indo-europee, alle greco-romane, per finire con gli elementi più prossimi, integranti la complessa e magistrale struttura politico-giuridica dell'Orbe Romano.

Per ciò che riguarda la comune formazione cristiana, non vi è dubbio che essa fu uno dei grandi e decisivi agenti di formazione dell'Impero, specialmente da Constantino in poi; ma non è meno vero che nella Penisalo Iberica questo componente attuò in modo particolarmente sostanziale nella formazione, soprattutto interna e costituzionale, delle sue molteplici nazioni (Asturia, Leone, Galizia, Algarves, ecc.).

Non sarebbe neppure necessario parlare, tale ne è l'evidenza, di quello che si può considerare come la co-partecipazione, più che l'influenza, gotico-mora.

I Goti dominarono fino al 711, cioè fino all'assassinio di WitTisa, ma la loro presenza non scomparve con il dominio mussulmano. E mentre la politica araba cominciò ad influire decisamente sul Diritto Pubblico, il Fuero JuzGn continuò a rimanere in vigore nel Diritto Privato dei popoli che preparavano la Riconquista e nelle stesse strutture moroiberiche. 
Per quanto riguarda il Diritto Canonico, la sua influenza informò tutti gli aspetti della vita peninsulare, e posteriormente quelli della Spagna e del Portogallo, già definiti come stati sovrani unificati.

Finalmente, denominiamo continente iberico strictu sensu quell'immenso complesso di elementi comuni che, provenendo dai Fenici, dai Celti e dai Cartaginesi, arriva fino a quella fase di maturamento che, nel secolo xir facilitò la formazione delle due grandi nazioni peninsolari. Durante il corso di otto secoli, essi le condussero per cammini simili, permenttendogli di conservare gli stessi caratteri fondamentali, forse come non avvenne per nessun altro popolo europeo, dato che esse serbano, ben radicati, i loro costumi, le loro tradizioni, la loro lingua che - castigliano o portoghese - è essenzialmente la stessa.

Da qui il fenomeno di certi documenti giuridici, rimasti in vigore a lungo e indistintamente, nel corso di molti secoli, in Portogallo e in Spagna. Per limitarci agli esempi più importanti, citiamo il Fuero Juzgo, il Fuero Real, e le Siete Partidas.

Con la scoperta del Nuovo Mondo, in cui i paesi iberici ebbero una parte assolutamente preponderante, questi elementi furono trasmessi direttamente all'America Latina, consolidandosi con la vigenza delle ordinanze di D. Filippo.

3. Le radici del Diritto Privato dell'America Latina. Evidentemente è nella stessa affinità sostanziale del Diritto dell'America Latina che troviamo le radici comuni del Diritto Privato.

Possiamo, tuttavia, precisarle e specificarle.

Si trovano: A) nel Diritto Romano, particolarmente in Giustiniano; в) nel Diritto Gotico, attraverso il Forum Judicum; c) nel Diritto Canonico, in modo speciale nelle costituzioni dei Papi dell'Alto Medioevo; p) nel Diritto Medioeva- 
le; E) nel Diritto Iberico specifico; F) nelle Ordinanze Filippine; G) nel Diritto Francese e in quello Germanico; H) nell'evoluzione intrinseca del Diritto Privato dell'Americat Latina.

A) Diritto Romano. Il Digesto, le Istituta, il Codice e le Novelle di Giustiniano hanno sempre ispirato il Diritto Privato dell'America Latina, e non solamente durante la fase coloniale; anche dopo che i diversi paesi del Nuovo Mondo ebbero conquistato l'Indipendenza, essi hanno contribuito all'elaborazione dei dispetti Codici. La stessa cosa avvenne con alcune opere complementari, come il Codice Teodosiano, le Sentenze di Paulo, e le Regole Singolari di Ulpiano.

в) Diritto Gotico. E stato valido in Portogallo fino al 1446, data delle Ordinanze di D. Alfonso v, e in Spagna fino al $10^{\circ}$ maggio del 1889 (fine del secolo xrx), data in cui è entrato in vigore il Codice Civile Spagnolo. Non è necessario mettere in evidenza la sua influenza sulla formazione generale del Diritto Privato delle nazioni la cui cultura proviene direttamente dalla Spagna e dal Portogallo.

c) Diritto Canonico. Le Decretali di Gregorio ix e iI Sexto di Bonifácio viII rappresentarono, senza dubbio, il momento più alto del Diritto Canonico Medioevale. Il Diritto Canonico era in vigenza all'inizio della formazione. dei popoli dell'America Latina; si mantenne in vigore durante le ordinanze di $\mathrm{D}$. Filippo e si addentrò nella vita dei nuovi paesi indipendenti, soprattutto nel campo del Diritto di Famiglia.

D) Diritto Medioevale generale. Da un lato i grandi testi del Diritto Positivo; dall'altro, e al di sopra di essi, gli insegnamenti dei Dottori. Per i primi basta citare i Libri Feudorum e le costituzioni di Lotario; per i secondi, la Glossa e la Post-Glossa, con Bartolus e Baldus.

Se è vero che quelle norme ebbero un'influenza minore delle altre, nonostante il loro significato altamente notevole, è pur vero che la lezione dei Dottori si costituì in Diritto 
Positivo, secondo il testo esplicito delle Ordinanze di D. Filippo.

E) Diritto Iberico Specifico. Si tratta in special modo del Fuero Real e delle Siete Partidas. Vi si può aggiungere, ancora, come molto importante, il Fuero de Cuenca, primo codice iberico della Riconquista (16 Gennaio 1 190), e il proprio Diritto del Foro locale, la cui indole conferma l'unità culturale intrinseca di tutta la Penisola Iberica.

Tutti questi testi fanno parte, innegabilmente, delle radici del Diritto Privato dell'America Latina, a volte per ciò che riguarda i contratti, o le eredità, a volte per ciò che riguarda, per esempio, il diritto di vicinanza.

F) Ordinanze Filippine. Siamo già nell'Età Moderna, ma bisogna mettere in evidenza il fatto che queste ordinanze costituirono il primo codice dell'Integrazione del Diritto Privato dell'America Latina, ancora prima dell'Indipendenza dei paesi del Terzo Mondo.

Da una parte, esse mantennero vive le più antiche tradizioni del Diritto Iberico, per mezzo di disposizioni varie che menzionavano espressamente il Diritto Romano, quello Canonico e la Dottrina dei Glossatori; dall'altra, essendo vigorate come ordinamento unico non solo in Spagna e in Portogallo, ma anche nelle rispettive colonie, esse prepararono non solamente il Diritto interno fino all'Indipendenza, ma continuarono a rappresentare il diritto positivo, in senso generale, fino alla promulgazione dei rispettivi codici. In Brasile, come è già stato detto, le Ordinanze di D. Filippo vigor arono fino al $1 .^{\circ}$ gennaio del 1917.

G) Diritto Francese e Diritto Tedesco. La maggior parte degli autori, nell'esposizione di motivi (v. per es. Mensage, di Montt e Ovalle al Congresso del Cile del 1855; l'esposizione di NarvaJa, al Governatore Provvisorio dell'Uruguai, nel 1867 , ecc.) rende noto chiaramente che, per l'elaborazione degli ordinamenti dell'America Latina, fu utilizzato il Codice di Napoleone. 
Ciò nonostante, non fu irrilevante il componente tedesco, da cui prese forma l'Esboco di Teixeira de Freitas in Brasile, e il Progetto SARsfield, in Argentina.

H) Evoluzione intrinseca del proprio Diritto Privato dell'America Latina. Nel sopra citado Messaggio, di Monit e O1 ( . .5, si trova un brano molto significativo, riguardo all'originalità del Progetto Cileno: “Desde luego concebiveis que no nos hallabamos en el caso de copiar a la letra ninguno de los códigos modernos. Era menester servir-se de ellos sin perder de vista las circunstancias peculiares de nuestro pais. Pero en lo que estas no presentaban obstaculos reales, no se ha trepedado en introducir provechosas inovaciones".

In realtà, questo schiarimento sarebbe valido per tutte le esposizioni di motivi dei codici dell'America Latina.

Tuttavia bisogna aggiungere che le innovazioni stesse come, per esempio, l'abolizione della restitutio in integrum, sono in certo qual modo comuni a questi codici, o per lo meno a varii gruppi di essi (per es. il Codice Argentino, Uruguaiano, Paraguaio, Brasiliano) in virtù dell'analogia nella sostanza degli elementi socio-giuridici delle rispettive culture.

4. Il Diritto delle Obbligazioni e dèi Contratti dell' America Latina. Tra i varii componenti delle radici comuni del Diritto Privato dell'America Latina, credo che debbano essere messi in risalto $i$ seguenti: A) componente romantico; B) visigotico; C) filippino; francorgermanico.

Il componente romanico, che basa i suoi fondamentali già sul nexum e sulla sponsio del Periodo del Jus Civile, arricchito dal Jus Gentium durante il periodo Aureo, influi più concretamente attraverso le consolidazioni giustinianee. Tuttativa non si possono sottovalutare nè il valore dottrinario del Libro III, delle Istitute di GAIo, dal Paragrafo 88 nè gli elementi del Codice Teodosiano, dato che entrarono a far parte integrante dei testi del Diritto Visigotico. 
D'altra parte, è necessario notare che il Diritto Giustinianeo fu presente nel Diritto Iberico, in modo diretto, solamente durante l'Alto Medioevo, e perciò, dopo la Riconquista.

Però non esiste alcun dubbio che egli constitui la più antica e solida base comune del Diritto delle Obbligazioni dell'America Latina.

Quanto al Diritto Visigotico, la sua maggior espressione in relazione al Diritto Iberico, e di conseguenza a quello dell'America Latina, è il Fuero Juzgo.

Rappresentando l'unificazione del Codice Euriziano (466-484) con il Breviario di Alarico II (506), promulgato da Flavio Recesvindo nel 671, durante il Settimo Concilio di Toledo, come è stato detto, esso vigorò in Portogallo fino alla metà del secolo $\mathrm{xv}$, e in Spagna fino alla seconda metà del secolo xix.

Come si può giudicare dai due testi fondamentali (latino-barbaro e espagnolo antico - v. Ed. della Reale Accademia Spagnola, del 1815) il rispettivo regolamento delle Obbligazioni e dei Contratti è meno sviluppato di quello dei precetti romani, ma porta già con sè il risultato di un altro sistema di vita, quello del Mondo Medioevale, del Renascimento e della stessa cultura contemporanea.

Per ciò che riguarda il Codice di D. Filippo, del 1603, essendo questo il primo testo dell'integrazione iberica, lo $\mathrm{fu}$, di conseguenza, anche per l'America Latina coloniale.

Questa integrazione riguardò più particolarmente il Diritto Privato che quello Pubblico, poichè, nonostante l'unificazione della corona spanhola e portoghesa in una sola, le respettive amministrazioni conservarono una certa autonomia, tanto che si potrebbe persino parlare di due corti, quella di Madrid e quella di Lisbona.

E non vi è bisogno di dire che nell'ambito del Dirito Privato, l'integrazione maggiore ebbe luogo nel campo del Diritto delle Obbligazioni, soprattutto a causa delle relazioni tra i mercanti delle colonie e quelli della metropoli. 
Per concludere, il Diritto Franco-Germanico fu influenzato, da un lato, dalla presenza del Codice di Napoli, dalla cristallizzazione effettuata, da PoTHIER Traité des Obligations, v.9, Oeuvres, II, Paris, 1861); dall'altro, dalla dottrina dei neo-pandettisti, come Savigny, Puchta, Hering, Dernburg, Windscheid, Kohler e tanti altri. Cosi forte fu il messaggio del pensiero giuridico tedesco, che il primo grande trattato sistematico francese, quello di Aubry e RAU fu compilato "secondo il metodo di ZacchariaE".

Così, come si può giudicare fin dai primi confronti stabiliti dell'immensa e proficua opera divulgatrice di RAOUL DE la Grasserie, e secondo quanto viene confermato nel Progetto stesso di Cosentrni, il Diritto delle Obbligazioni e dei Contratti dell'America Latina è sostanzialmente lo stesso, tanto da far propendere verso un'unificazione, i cui benefici dovranno farsi sentire, non solo nella comunità del Terzo Mondo, ma anche e principalmente, nell'equilibrio delle relazioni sociali, economiche e politiche in campo internazionale.

V. Prospettive circa Un’Unificazione Universale del Diritto.

1. L'America Latina e l'America Anglosassone.

2. L'America Latina e l'Europa. 3. L'America $e$

l'Oriente. 4. L'America Latina e il Mondo Africano.

1. L'America Latina e l'America Anglosassone. Chiamiamo America Anglosassone l'insieme delle nazioni e dei popoli americani che, come dice l'espressione, contengono, nell'origine e nella cultura, forti elementi nordici. Per esempio: gli Stati Uniti, il Canadà, il Suriname, Trinità, Tobago, le Barbados, le Bahamas, ecc.

Varii fattori rendono possibile l'unificazione graduale del loro Diritto delle Obbligazioni, a quello dell'America Latina. 
Le relazioni politiche e commerciali dell'America Latina con gli Stati Uniti hanno un'importanza preponderante. Di fatto, in realtà, se, da una parte, fu l'indipendenza nord-americana a provocare quella di tutti gli altri paesi del Nuovo Continente, e se la Dichiarazione di Monroe servi a tutti come scudo comune, come elemento equilibrante nelle relazioni con l'Europa della Santa Alleanza, dall'altra parte, nessuno ignora l'esistenza di una massa di investimenti economici che, a poco a poco, vanno incrementando la possibilità, la necessità, anzi, di servirsi di un diritto il più possible unificato.

Per citare un esempio, il "leasing" che è un tipo di contratto oriundo dal "Common Law", circola francamente nel sistema consuetudinario e giuridico dei paesi dell'America Latina.

Inoltre, la ricchezza delle origini del nostro Diritto, in cuí non manca, come abbiamo visto, l'elemento nordico, presenta punti di contatto veramente fondamentali. In base a questi è possibile porsi una prospettiva, simile, riguardo agli altri popoli americani in questa stessa area.

2. L'America Latina e l'Europa. Se consideriamo unificazione del nostro Diritto in relazione all'Europa, vediamo che non sono solamente gli elementi iberici, già messi in risalto nei due Congressi Ispano-Luso-Americani del Diritto Internazionale ad avere un'importanza rilevante (v. Oscar Martins Gomes, A Possibilidade da Unificação do Direito Privado Hispano-Luso-Americano, in Atas II, pg. 392, São Paulo, 1957).

Oltre a questi elementi, dobbiamo considerare le sopracitate origini giuridiche comuni, di natura franco-germanica, canonica e greco-latina.

La loro stessa forma, proveniente dal Diritto Latinoamericano, lungi dal rappresentare un fattore di allontanamento, se ci si riferisce ai sistemi europei, è causa, piuttosto, 
di avvicinamento, per tutta la contribuizione che può offrire al progresso tecnico del Diritto del vecchio Continente.

Basta ricordare che l'Esboco di TelxeIra de Freitas, ispiratore dei Codici dell'Argentina e dell'Uruguai, antecedette di quattro decadi il sistema fondamentale del Codice Tedesco. D'altra parte, si moltiplicarono gli esempi, come quello del Codice del Panamà, art. 1217, che, trattando di beni immobili, in materia di vendita di beni di terzi, restringe detta vendita al venditore purchè questi sia in possesso dél giusto titolo; Cosentini, nel suo Progetto, lo presenta come "une exception justifée" (pag. 13), adottada da lui come testo.

D'altra parte, l'Europa stessa, spinta dal Mercato Comune, testimonia la necessità dell'unificazione giuridica, come infrastruttura dell'integrazione, il che rende più facile estendere questa prospettiva all'America Latina c viceversa (v. p. es. il Projet d'Unification de la Société Anonyme, del "Conseil des Communautées Européennes", che crea la "Societas Europea - S. E.").

3. L'America Latina e l'Oriente. In questo campo agisce, in modo decisivo, il fattore demografico e culturale, a causa del numerosissimo nucleo di cittadini latino-americani di origine indiana, coreana, cinese, e specialmente giapponese in essa esistente.

Sebbene l'assimilazione sia integrale, essi costituiscono un elemento innegabile e prezioso per il dialogo fra l'America Latina e l'Oriente.

A parte questo, il grande sviluppo dei mezzi di comunicazione ha accorciato le distanze, ha chiarito molte situazioni, ha incentivato l'intercambio delle informazioni, in modo da facilitare la conoscenza di dati sicuri per $\mathbf{i}$ grandi investimenti orientali nei paesi dell'America Latina.

Il nucleo maggiore è costituito dai giapponesi, ma la Corea stessa sta già stringendo le sue relazioni commerciali, mentre le prime missioni economiche dei paesi dell'Ame- 
rica Latina studiano in loco le possibilità di un'intercambio attivo e operante con la Cina Continentale.

4. L'America Latina e il Mondo Africano. ì sufficiente ricordare che le origini della Storia e dell'Economia dell'America Latina sono legate all'elemento negro.

Lasciando da parte le condizioni iniziali della sua integrazione, il Negro, oriundo dall'Africa, costituisce una delle travi maestre di tutta la Cultura latino-americana, soprattutto perchè, tra di noi, è arrivato a godere della più assoluta uguaglianza giuridica e sociale.

Di consequenza, la popolazione dell'Ameria Latina è anche, in gran parte, formata da gente di sangue, d'indole e di cultura africana.

Le lingue parlate tra noi sono farcite di parole non solo di origine africana ma africane vere e proprie, che si sono continuate ad usare e sono entrate a far parte del miglior linguaggio.

Nell'America Latina si professano religioni africane che, lungi dall'estinguersi, sono soggette, periodicamente, ad un incremento considerevole, dovuto alla facile acclimatazione al nostro ambiente.

La nostra cucina, quando veramente raffinata, lo è a ragione dell'influenza africana.

E nelle arti, punto sublime dell'anima di un popolo, l'Africa è sostanzialmente presente: nel ritmo, nella melodia e nella tematica stessa della musica; nella coreografia delle danze; nella plasticità della scultura; nei colori forti e vivi di una pittura ricca ed espressiva.

Non è necessario esaltare l'importanza di questi elementi per stabilire il dialogo; questo senza parlare dei popoli africani che hanno subito una maggior influenza europea, la cui fisionomia è interamente analoga alla nostra.

Cosicchè l'unificazione del Diritto afro-latino-americano, in relazione a quelli, ha come sostrato le affinità del 
sangue e della cultura; in relazione a questi, contiene in più altri due elementi: la formazione giuridica mediterranea e la visione, nuova e progressista, di questa formazione.

Oltre a ciò, i contratti diplomatici e commerciali cơn paesi come il Kenia e il Senegal, il Togo e l'Etiopia, fanno credere alla possibilità di questa unificazione in un futuro ogni volta più prossimo.

\section{Conclusione.}

1. Visione d'insieme. 2. Fallimento delle unificazioni europee passate. 3. L'America Latina come il cammino più accessible per un' unificazione del Diritto. 4. L'unificazione del Diritto delle Obbligazioni e dei Contratti come punto di partenza.

1. Visione d'insieme. Secondo quanto abbiamo visto nel corso di questa esposizione, l'oggetto del Diritto Internazionale è il Bene Comune della comunità delle nazioni, e soprattutto dell'essere umano nell'ambito di questa comunità; da qui la sua evoluzione graduale, in vista di questo desiderato, raggiunta, nei tempo attuali, con la fusione dei paesi in una società internazionale, dalla strutura giuridicamente organica.

Come complemento di questa società, e con lo scopo di coadiuvare al raggiungimento di questa sua specifica finalità, sono sorte le entità plurinazionali, tra cui il Mercato Comune Europeo.

D'altro canto, al formarsi dello Stato Moderno, si è creata la rottura di una certa unità del Diritto Consuentudinario Internazionale, che ha suscitato l'interesse per un ritorno a questa unità giuridica, indispensabile all'equilibrio economico e politico della Soceità delle Nazioni.

Nell'evoluzione del Diritto Internazionale moderno, l'America Latìna è stata sempre presente e ha sempre attuato, fin dai primi anni dell'indipendenza dei suoi paesi, come un blocco omogeneo. 
Questa realtà ha reso possibile la sua gradativa determinazione, che, tuttavia, per consolidarsi, dipende dall'integrazione delle sue istituzioni giuridiche.

L'integrazione giuridica dell'America Latina non solo è di grande importanza per il diritto universale, ma è perfettamente fattibile a causa di varii e significativi fattori: l'affinità sostanziale dei rispettivi sistemi nel campo del Diritto Privato, e in modo particolare in quello delle Obbligazioni e dei Contratti, l'identità, o per lo meno l'analogia, delle cause efficienti.

D'altra parte, l'America Latina presenta caratteristiche etnografiche, sociali e giuridiche, dalle quali defluiscono relazioni particolarmente speciali con l'America Anglosassone, l'Europa, l'Oriente e il Mondo Africano.

2. Il fallimento delle unificazioni europee passate. Cosentini, parlando del Progetto Francese-Italiano, così si esprime: “À vrai dire, le projet franco-italien fut plutôt une déception pour le monde scientifique. On s'attendait à quelque chose de plus complexe, de plus organique, ̀̀ un ensemble de reformes plus accentuées, dans un domaine ou la doctrine et la pratique avaient manifesté des tendences fortement révisionnistes". D'altra parte, fa notare che - "Il ne faut d'ailleurs pas oublier que la tâche essentielle de la commission franco-italienne était d'harmoniser la législation positive des deux pays" (pág. 6).

Di qui la ragione del suo progetto di un Code International des Obligations nel quale, contro i 739 articoli della sua opera precedente, ne furono proposti 3.115, che, sviluppati, sommano a 5800 .

Ma se il Progetto Francese-Italiano non entrò in vigore neppure nei rispettivi paesi, probabilmente a causa di un eccesso di nazionalismo, non dei giuristi che lo compilarono, ma degli organismi dirigenti, e per la carenza di attualità scientifica, il Progetto di Codice Internazionale delle Obbligazioni dovette affrontare per lo meno due osta- 
coli fondamentali: l'eccesso di disposizioni troppo vincolanti, e l'impermeabilità del mondo europeo alle innovazioni, molte delle quali chieste imprestito all'America Latina, che non fossero esclusivamente un prodotto del loro ambiente culturale.

3. L'America Latina come il cammino più facile verso l'unificazione del Diritto. A questa mancanza di universalità nelle culture europee, che, oltre ad essere esclusive in relazione al resto del mondo, sono, in definitiva, esclusive anche dentro i loro limiti (basta ricordare il numero infinito di dialetti e persino di lingue diverse parlate nell'ambito di territori abbastanza ristretti) - fa riscontro un parallelo opposto nell'America Latina, dove praticamente si parla una sola lingua la cui origine storica è assolutamente la stessa, così come sono basicamente gli stessi il suo Diritto e il suo processo evolutivo.

D'altro lato, a questo si deve aggiungere l'universalità della formazione etnografica e culturale in relazione alle altre parti del mondo, secondo quanto abbiamo già fatio notare anteriormente.

Ancora una volta, quindi, appare chiaramente la tendenza all'Internazionalità dell'America Latina alla quale, tutto ce lo fa credere, è affidata la missione di rappresentare il primo cammino effettivamente possibile verso l'unificazione universale del Diritto, passo indispensabile per un appianamento delle relazioni tra i popoli.

4. L'unificazione del Diritto delle Abbligazioni e dei Contratti dell'America Latina come punto di partenza. Varii fattori ci portano a credere ad una unificazione del Diritto delle Obbligazioni e dei Contratti dell'America Latina, come punto di partenza per una unificazione universale del Diritto.

Tra gli altri, come è già stato segnalato, possiamo indicare la sua intrinseca maturità, l'influenza esercitata sulla struttura tecnica dei sistemi di altri popoli e soprattutto, la 
possibilità della sua stessa unificazione, data l'affinità dei suoi codici, (come già è stato dimostrato) affinità che non presenta in altre società di nazioni, altrettanti punti di contatto cosi ampi e significativi.

Se tutto ciò è vero nel campo del Diritto, e particolarmente del Diritto Privato, assume una veridicità ancora più lampante nell'ambito del Diritto delle Obbligazioni e dei Contratti.

D'altra parte, la sua esecuzione non solo può, ma deve essere immediata.

Può essere immediata, perchè da tale diritto risultano chiari e rapidamente dimostrabili, gli elementi della sua massima e naturale messa in opera. Deve essere immediata, in quanto costituisce uno degli elementi basici ai quali fa appello l'integrazione dell'America Latina: tra gli altri, principalmente l'indispensabile istituzione di un suo Mercato Comune.

Altri due orizzonti, ancora più vasti, dovranno aprirsi di fronte a questo provvedimento: il già menzionato passo iniziale verso l'unificazione universale del Diritto e la fusione dei rispettivi diritti dei paesi dell'America Latina in un blocco omogeneo, nell'ambito delle nazioni e degli organismi plurinazionali, tra i quali il Mercato Comune Europeo. 